DOI: https://doi.org/10.11144/Javeriana.upsy18-4.apas

\title{
Aplicación de un programa de aprendizaje socioemocional y moral para mejorar la convivencia en educación secundaria*
}

\section{Application of a Socioemotional and Moral Learning Program for Promoting Healthy Relationships in Secondary Education}

\author{
FRANCESC SiDERA ${ }^{\mathrm{a}}$ \\ Universitat de Girona, España \\ ORCID: http://orcid.org/0000-0001-7107-0450 \\ Carles Rostan \\ Universitat de Girona, España \\ ORCID: http://orcid.org/0000-0003-4223-0368 \\ JoRdi Collell \\ Práctica Privada, España \\ ORCID: http://orcid.org/0000-0002-8913-2581 \\ SARA Agell \\ Universitat de Girona, España \\ ORCID: http://orcid.org/0000-0002-4215-8442
} a Autor de correspondencia. Correo electrónico:
francesc.sidera@udg.edu

Para citar este artículo: Sidera, F., Rostan, C., Collell, J., \& Agell, S. (2019). Aplicación de un programa de aprendizaje socioemocional y moral para mejorar la convivencia en educación secundaria. Universitas Psychologica, 18(4), 1-14. https://doi.org/10.11144/Javeriana.upsy18-4.apas

\section{RESUMEN}

Se llevó a cabo una intervención educativa en 2.ํ curso de secundaria para mejorar la convivencia y reducir la agresividad. Los 64 participantes (media de edad $=13.57$ años; $D E=0.35$ ), divididos en un grupo control y otro de intervención, respondieron a un pretest y un postest para evaluar el cambio en el clima escolar, la empatía, la desconexión moral y la agresividad. Se realizaron 11 sesiones de intervención grupales para trabajar las habilidades socioemocionales y morales del alumnado. Los resultados muestran una correlación negativa entre la desconexión moral y la agresividad total en el pretest $(r=-0.526 ; p<0.001)$ y el postest $(r$ $=-0.463 ; p<0.001$ ). Además, existió una correlación entre el cambio observado en la agresividad impulsiva y el cambio en la subescala de fantasía de la empatía $(r=-0.326 ; p=0.01)$. Sin embargo, la intervención no fue efectiva para reducir la agresividad ni para mejorar la empatía o la desconexión moral. Además, empeoró el clima escolar en ambos grupos (pretest: $Z=-2.132 ; p=0.033$; postest: $Z=-3.473$; $p<0.001$ ). Finalmente, se discuten las implicaciones para el diseño de programas de intervención socioemocionales y morales.

Palabras clave

convivencia; agresividad; educación secundaria; clima escolar; empatía; habilidades socioemocionales.

\section{ABSTRACT}

We carried out an educational intervention in the 2 nd course of secondary school with the aim of improving healthy relationships and reduce aggressive behaviors in the classroom. The 64 participants (mean age $=13.57$ years; $S D=0.35$ ) were divided in a control group and an intervention group, and they responded to a pretest and a posttest in order 
to evaluate the change in school climate, empathy, moral disengagement and aggressiveness. The educational intervention consisted of 11 group sessions directed at improving students' socioemotional and moral skills. The results showed a negative correlation between moral disengagement and total aggressiveness both in pretest $(r$ $=-0.526 ; p<0.001)$ and postest $(r=-0.463 ; p<0.001)$. Furthermore, a negative correlation existed between the change observed in impulsive aggression and the change in the fantasy subscale of empathy $(r=-0.326 ; p=$ 0.01). However, the intervention was not effective in reducing aggressiveness or in improving empathy or moral disengagement. Furthermore, school climate decreased in both groups (pretest: $Z=-2.132 ; p=0.033$; postest: $Z$ $=-3.473 ; p<0.001)$. Finally, the implications for the design of socioemotional and moral intervention programs are discussed.

Keywords

healthy relationships; aggressiveness; secondary school; school climate; empathy; socioemotional skills.

El objetivo general de este estudio fue llevar a cabo y evaluar un programa de intervención educativa basado en el aprendizaje socioemocional y la educación moral, con el fin de promover la convivencia en el aula y prevenir y reducir la agresividad en los centros de Enseñanza Secundaria.

La agresividad en la infancia y la adolescencia puede tener trágicas consecuencias tanto para la víctima como para el perpetrador, así como para la escuela, la familia y la sociedad. Las víctimas de conductas agresivas, aparte del dolor físico y/o psicológico, pueden sufrir rechazo de sus compañeros, soledad, desórdenes internalizantes, sentimiento de incompetencia e inadaptación a la escuela. Si la agresividad es persistente, a los problemas anteriores se les puede añadir ansiedad social, depresión, ideación suicida, absentismo escolar y consumo de sustancias (Card, Stucky, Sawalani, \& Little, 2008; Hawker \& Boulton, 2000). Según un informe realizado por el Hospital Sant Joan de Déu de Barcelona, las conductas disociales y disruptivas en la infancia y la adolescencia suponen una de las demandas más importantes de los centros de salud mental (Matalí, 2016). Por otra parte, un número pequeño pero significativo de niños y niñas que empiezan pronto a mostrar comportamientos agresivos se convertirán en delincuentes persistentes a lo largo de su vida (Herrenkohl, Kosterman, Mason, \& Hawkins, 2007), del mismo modo que la victimización violenta en los niños es un factor de riesgo de conductas delincuentes a lo largo de toda la vida (Weir \& Kaukinen, 2015).

La literatura científica actual distingue entre agresividad proactiva y reactiva, ya que estos constructos son diferentes tanto en la infancia como en la edad adulta. Mientras que la agresividad proactiva (o también instrumental, o no impulsiva) persigue un propósito o un hito (está asociada con recompensas), y es por lo tanto, premeditada y faltada de provocación; la agresividad reactiva (o impulsiva) se refiere a comportamientos no planificados y espontáneos, que son a menudo respuestas a amenazas (reales o percibidas) o frustraciones (Blair, 2003; Rieffe et al., 2016). Además, estos tipos de agresividad se han relacionado con distintos perfiles comportamentales y neurocognitivos (Carroll, McCarthy, Houghton, Sanders O'Connor, \& Zadow, 2018). A pesar de esta distinción, debemos tener en cuenta que algunos estudios indican correlaciones entre ambos tipos de agresividad, y por tanto, que muchos niños se involucran en ambos tipos de comportamientos (Bookhout, Hubbard, \& Moore, 2018). Por otra parte, esta distinción se refiere a la función de la agresividad, mientras que otras categorizaciones tienen que ver con su forma, como, por ejemplo, la distinción entre la agresividad física y relacional, que están asociadas a aspectos diferentes del desarrollo (O'Toole, Monks, \& Tsermentseli, 2017).

Los centros educativos son un lugar privilegiado para la prevención de la conducta agresiva, y representan una oportunidad para cambiar el comportamiento social de los adolescentes (Greenberg et al., 2003). Sin embargo, los profesores consideran que los recursos que tienen en las aulas para afrontar las conductas disociales y disruptivas son deficientes (Matalí, 2016). Es en este marco que los programas de aprendizaje socioemocional y moral adquieren su significado. Esto implica aprender habilidades emocionales y sociales para interaccionar adecuadamente con los demás en 
diferentes contextos, practicar comportamientos saludables y comportarse con responsabilidad y respeto (Durlak, Weissberg, Dymnicki, Taylor, \& Schellinger, 2011).

En la línea anterior, un metaanálisis de más de 200 investigaciones evaluando iniciativas diseñadas para enseñar habilidades socioemocionales encontró evidencias de beneficios en aspectos como: a) habilidades socioemocionales (que incluía, por ejemplo, reconocimiento emocional, gestión del estrés, empatía o resolución de problemas); b) actitudes hacia uno mismo, los demás y la escuela; c) aumento de los comportamientos sociales; d) reducción de problemas de comportamiento y internalizantes y e) mejora en el rendimiento académico (Durlak et al., 2011). Otros metaanálisis posteriores confirman los posibles beneficios para los niños y adolescentes de los programas que enseñan habilidades socioemocionales (ver, por ejemplo, Taylor, Oberle, Durlak, \& Weissberg 2017; Wiglesworth et al., 2016). Así pues, para el éxito dentro y fuera de la escuela, es totalmente necesario que el sistema educativo incluya habilidades, no estrictamente académicas, orientadas hacia las habilidades socioemocionales, cognitivas y morales, sin las cuales la implicación y la motivación de los estudiantes hacia la escuela continuarían siendo deficitarias, especialmente para aquellos que más lo necesitan (ver DePaoli, Atwell, \& Bridgeland, 2017; Mahoney, Durlak, \& Weissberg, 2018; Weissberg, Durlak, Domitrovich, \& Gullotta, 2015).

Algunos autores consideran las habilidades socioemocionales como la "missing piece" de la escuela (Elias, 2009). Un aspecto clave para que el aprendizaje socioemocional deje de estar "desaparecido" de la escuela, es investigar para entender mejor cómo esta puede implementar programas efectivos y sostenibles que satisfagan las necesidades socioemocionales de los estudiantes. Más aún, cuando muchas de las investigaciones se han llevado a cabo en la educación infantil y primaria, quizá porque la educación secundaria está más focalizada hacia los resultados académicos, más que atender a los estudiantes como un todo.
En la actualidad, ya existen algunos programas universales que se han mostrado eficaces para la reducción de la agresividad y el acoso en estudiantes de secundaria, tales como el Olweus Bullying Prevention Program (Olweus, 1991; Olweus, Limber, \& Breivik, 2019) o el Kiva (Salmivalli, Kärnä, \& Poskiparta, 2011; ver da Silva et al., 2017), pero este tipo de programas requiere de una gran implicación de toda la comunidad educativa, planteamiento que los centros educativos no siempre están en disposición o tienen los recursos para adoptar. Por este motivo, se ha diseñado una intervención que pueda ser llevada a cabo por los profesorestutores de los centros educativos de secundaria, de manera más focalizada. Esta intervención consiste en un programa de tutorías que tiene como objetivo principal mejorar la convivencia (mejorar el clima de aula y reducir las conductas agresivas y disruptivas) en las aulas de secundaria.

La intervención que se plantea en el presente estudio se basa en un modelo integrador, que incluye principalmente aspectos del aprendizaje socioemocional (Elias, Parker, Kash, Weissberg, \& O'Brien, 2008) y de la educación moral de los y las estudiantes. Basándose en los resultados de un amplio estudio, Conley (2015) concluye que los programas de aprendizaje socioemocional deberían incluir las siguientes competencias: a) auto-conciencia (reconocer los propios pensamientos y sentimientos, limitaciones, fortalezas, etc.); b) autocontrol (regular adecuadamente las propias emociones, pensamientos y comportamientos y ser capaz de alcanzar un bienestar emocional); c) conciencia social (comprender, respetar y empatizar con los demás, así como identificar apoyos sociales); d) habilidades para relacionarse (i. e., establecer y mantener relaciones saludables, saber pedir y ofrecer ayuda, etc.) y e) tomar decisiones responsables (saber decidir en base a la ética, responsabilidades, posibilidades para promover el bienestar propio y de los otros). Aparte de estos aspectos, la intervención que planteamos aquí también pretende incidir en el clima de aula y en las relaciones interpersonales. Naturalmente, el sistema de relaciones donde se desarrollan los chicos y chicas es muy complejo y abarca la 
familia y la comunidad, pero, como ya hemos dicho, nuestra intervención se enmarcará dentro de un plan de acción tutorial en el aula. Aunque hay datos que indican que los programas que incorporan múltiples componentes (por ejemplo, a toda la comunidad educativa o a las familias) pueden ser más eficaces, estos también tienen más dificultades de implementación que pueden afectar a su efectividad (ver Durlak et al., 2011).

El modelo del aprendizaje socioemocional pone énfasis en la importancia de la coordinación del afecto, el comportamiento, la cognición y el contexto de desarrollo para adquirir las habilidades y las actitudes necesarias para manejarse en los ambientes sociales. Por otra parte, la educación moral da una dirección y un sentido a las habilidades socioemocionales. En esta línea, se ha destacado la importancia de este tipo de programas para la prevención de la agresividad, la promoción de la salud, el desarrollo moral, el rendimiento y el compromiso académico (Conley, 2015; Durlak et al., 2011). Además, una revisión actual de Taylor et al. (2017) sobre el impacto de 82 programas de aprendizaje socioemocional en niños (desde educación infantil hasta la educación secundaria), muestra que el efecto de los programas revisados en siete categorías de resultados (habilidades socioemocionales, actitudes, comportamiento social positivo, resultados académicos, problemas de comportamiento, malestar emocional y uso de drogas) todavía era significativamente positivo (medidas del efecto medias entre 0.13 y 0.33 ) entre las 56 y las 195 semanas, en promedio (dependiendo del tipo de categoría evaluada), posteriores a la intervención.

Tomando como base el modelo teórico comentado anteriormente, se plantea una intervención educativa que incluye el trabajo de las habilidades socioemocionales (comprensión social, empatía, funciones ejecutivas $y$ regulación emocional), las habilidades morales (razonamiento y desconexión moral) y el clima escolar, con el objetivo principal de prevenir y reducir la agresividad en la etapa de educación secundaria (2. . de ESO). Por otra parte, los objetivos específicos de este estudio son la evaluación del efecto del entrenamiento en: a) algunas capacidades incluidas en el modelo de aprendizaje socioemocional (empatía y desconexión moral); b) el clima de clase y c) el comportamiento agresivo premeditado e impulsivo de los participantes. Finalmente, queremos estudiar la relación entre las distintas variables estudiadas, y si existen relaciones entre los cambios observados en estas variables entre el pretest y el postest.

\section{Método}

\section{Participantes}

Un total de 64 participantes fueron incluidos en este estudio ( 17 chicas y 15 chicos en el grupo control y 15 chicas y 17 chicos en el grupo de intervención). Los integrantes de esta muestra incidental provenían de cuatro clases de 2. de ESO de dos institutos públicos distintos, por lo que en cada instituto participó un grupo control y un grupo de intervención. Un instituto era de doble línea y el otro de triple. Debemos señalar que inicialmente participaron un total de 80 chicos y chicas, pero 16 de ellos fueron descartados por los siguientes motivos: a) un participante cambió de instituto durante el estudio; b) tres participantes respondieron aleatoriamente en el pretest y/o en el postest; c) tres participantes del grupo de intervención tuvieron una participación inferior al $50 \%$ de las sesiones y d) nueve participantes dejaron cuestionarios sin responder. Adicionalmente, sus familias fueron informadas acerca del estudio que se iba a llevar a cabo, y se obtuvo su consentimiento informado.

La edad media (calculada a partir de la fecha de inicio del pretest) fue de 13.57 años $(D E=$ $0.35)$, siendo esta de 13.62 años $(D E=0.31)$ en el grupo control y de $13.52(D T=0.39)$ en el grupo de intervención. Con la prueba de MannWhitney se comprobó que no existían diferencias significativas entre los dos grupos en cuanto a la edad $(p>0.05)$. Por otra parte, la misma prueba mostró que no existen diferencias entre los dos grupos en cuanto a razonamiento no verbal $(p$ 
$>0.05$; ver apartado de material), siendo las puntuaciones medias similares en ambos grupos (media del grupo control $=135.31, D E=12.97$; media del grupo de intervención $=133.55 ; \mathrm{DE}$ $=2.18$ ).

\section{Instrumentos}

Test de matrices del British Ability Scales ([BAS 2]; Arribas 8 Corral, 2011)

Este instrumento de evaluación solo fue empleado en el pretest, cuando se utilizó la subescala de matrices para obtener una medida de razonamiento no verbal. En esta prueba, se presentan matrices con cuatro o nueve celdas con una figura en cada una, y una figura en blanco en una de ellas. El participante debe elegir una de entre seis alternativas de respuesta. Se utilizó la puntuación de aptitud que tiene en cuenta el nivel de dificultad de los ítems aplicados a cada participante. El índice $\alpha$ de Cronbach en esta subescala se sitúa entre 0.82 y 0.89 , entre los 12 y los 14 años de edad.

Instrumentos de evaluación usados en el pretest y el postest

- Interpersonal Reactivity Index (IRI) (Davis, 1980; adaptación al castellano de Carrasco, Delgado, Barbero, Holgado, \& del Barrio, 2011)

Este instrumento evalúa la empatía y la aplicación se da en edades comprendidas entre los nueve y 16 años, y consta de 28 ítems divididos en cuatro subescalas y evaluados en escala Likert con cinco posibles respuestas. Davis (1980) informa que los coeficientes $\alpha$ de fiabilidad interna para cada una de las cuatro subescalas oscilaron entre 0.7 y 0.78 .

- Cuestionario de Agresividad Premeditada e Impulsiva ([CAPI-A]; Andreu, 2010)

Esta escala distingue dos tipos de agresividad, impulsiva y premeditada. La aplicación de la escala se da en edades comprendidas entre 12 y 17 años. El instrumento consta de 24 ítems evaluados en una escala Likert con cinco posibles respuestas y seis ítems con tipo de respuesta verdadero/falso. Además, el CAPI-A muestra una fiabilidad interna según $\alpha$ de Cronbach de 0.83 en la agresividad premeditada y de 0.82 en la agresividad impulsiva.

- Mechanisms of Moral Disengagement Scale (Bandura, Barbaranelli, Caprara, \& Pastorelli., 1996)

Evalúa la desconexión moral a través de 32 ítems en formato de escala Likert con tres posibles respuestas. De acuerdo con los autores, el coeficiente de fiabilidad alpha de esta medida es de 0.82 .

- Cuestionario "¿Cómo te va en el Instituto?” (Collell \& Escudé, 2004)

Este cuestionario es una adaptación del Cuestionario para estudiantes (Ortega, del Rey, \& Gómez, 2002), y evalúa la percepción del alumnado del clima escolar respecto la conflictividad, el entorno y el bienestar subjetivo. Consta de una escala Likert de 14 ítems con cuatro posibilidades de respuesta (nunca, a veces, a menudo o siempre), más una pregunta final de respuesta abierta.

- Cuestionario de datos sociodemográficos

Se utilizó un cuestionario, respondido por los tutores, en el que se pedía la siguiente información: sexo del participante, edad del participante y de los padres, nivel educativo de los padres, número de hermanos del participante y existencia de algún tipo de trastorno o discapacidad.

\section{Procedimiento}

En primer lugar, se contactó con algunos centros educativos de la provincia de Girona, exponiendo el proyecto que se quería llevar a cabo. Una vez se encontraron dos centros educativos interesados en participar, se realizó una reunión en cada centro con los tutores de las aulas implicadas, y se les pidió que llenaran las hojas de datos. Antes de empezar el estudio, se identificó al alumnado con un código. A continuación, se aplicó el pretest, que se administró de manera colectiva y se dividió en dos sesiones de 60 minutos cada una. Durante estas dos sesiones los estudiantes respondieron 
a los cuestionarios, que fueron administrados por los autores del estudio. La semana después de terminar el pretest, se inició la intervención educativa. Esta consistió en 11 sesiones de una hora, a razón de una sesión por semana, dentro del horario de tutorías, donde se trabajaron los aspectos detallados en la Tabla 1. Las sesiones de intervención se llevaron a cabo por parte de los miembros del equipo de investigación. Los tutores de cada grupo (o algún otro profesor en caso de que el tutor no pudiera), estuvieron presentes en todo momento durante las sesiones de intervención, sin tener un papel activo en la acción educativa, y solo interviniendo puntualmente para regular el comportamiento del alumnado. Una vez finalizada la intervención se realizó el postest con el mismo procedimiento con el que se llevó a cabo el pretest, y con las mismas pruebas, a excepción de la prueba de matrices del BAS-2, que solo se administró en el pretest.

\section{Tabla 1}

Descripción de las sesiones de intervención

\begin{tabular}{|c|c|c|}
\hline sión & Aspectos trabajados & Objetivos y actividades \\
\hline \multirow[b]{2}{*}{1} & $\begin{array}{l}\text { Clima de } \\
\text { de conviv }\end{array}$ & $\begin{array}{l}\text { Objetivo: Reflexionar sobre el clima del aula, las normas de convivencia y nuestras } \\
\text { relaciones interpersonales. }\end{array}$ \\
\hline & $\begin{array}{l}\text { Relaciones } \\
\text { interpersonales }\end{array}$ & $\begin{array}{l}\text { Actividades: 1) Debate sobre el clima, las normas de clase y sus valores } \\
\text { Dinámica sobre cómo nos tratamos con otras personas. }\end{array}$ \\
\hline \multirow{2}{*}{2} & $\begin{array}{l}\text { Concepto de } \\
\text { agresividad }\end{array}$ & \multirow{2}{*}{$\begin{array}{l}\text { Objetivo: Reflexionar sobre el concepto de agresividad, } \\
\text { Actividades: I) Debate sobre la agresividad (tipos, causas, justificaciones, } \\
\text { consecuencias y difierencias individuales;) 2) Compartir vivencias de situaciones } \\
\text { donde han sido agresores, victimas u observadores y sus emociones en las mismas. }\end{array}$} \\
\hline & $\begin{array}{l}\text { Compartir vivencias de } \\
\text { situaciones agresivas }\end{array}$ & \\
\hline & Conciencia emocional & $\begin{array}{l}\text { Objetivo: Identificación de emociones en distintas situaciones. } \\
\text { Actividades: 1) Dinámica de relaciones interpersonales ("icómo }\end{array}$ \\
\hline 3 & $\begin{array}{l}\text { Emociones im } \\
\text { en situaciones } \\
\text { agresividad }\end{array}$ & 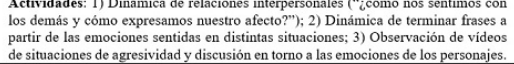 \\
\hline \multirow[b]{2}{*}{4} & $\begin{array}{l}\text { Estrategias de } \\
\text { regulación }\end{array}$ & $\begin{array}{l}\text { Objetivo: Aprender estrategias para regular las emociones, en especial en } \\
\text { situaciones de agresividad. }\end{array}$ \\
\hline & $\begin{array}{l}\text { regulaci } \\
\text { emocio }\end{array}$ & $\begin{array}{l}\text { Actividades: 1) Teatralización y discusión grupal de situaciones de regulación de } \\
\text { emociones negativas; 2) Valoració en grupos de estrategias para la regulación de } \\
\text { emociones propuestas por los investigadores y búsqueda de ejemplos de las mismas. }\end{array}$ \\
\hline \multirow[b]{2}{*}{5} & Funciones ejecutivas & \multirow{2}{*}{ 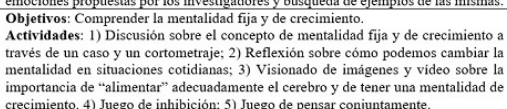 } \\
\hline & $\begin{array}{l}\text { Mentalidad fija y } \\
\text { mentalidad de } \\
\text { crecimiento }\end{array}$ & \\
\hline 6 & $\begin{array}{l}\text { Compor } \\
\text { asertivo }\end{array}$ & 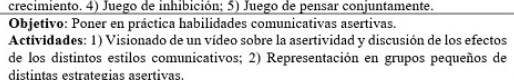 \\
\hline 7 & $\begin{array}{l}\text { Resoluci } \\
\text { conflicto }\end{array}$ & $\begin{array}{l}\text { as. } \\
\text { re el concepto de conflicto y sobre las estrategias que } \\
\text { resolución de conflictos y discusión de las distintas } \\
\text { sibles; 2) Refflexión sobre los puntos de vista en los } \\
\text { nes ambiguas. }\end{array}$ \\
\hline 8 & Sesgos atribucionales & $\begin{array}{l}\text { conflctost a partit de magent } \\
\text { Objetiv: Tomar conciencia } \\
\text { Actividades: 1) Discusuion } \\
\text { ambigua; 2) Juego de resoluc }\end{array}$ \\
\hline 9 & Desconexión & $\begin{array}{l}\text { Objetitiv: Tomar conciencia de los mecanismos de desconexión moral y de su uso } \\
\text { para evitar emociones negativas. } \\
\text { Actividades : I) Visionado y discusión de un video de obediencia a la autoridad; } 2) \\
\text { Discusión sobre distintas estrategias de desconexión moral en una situación de acoso } \\
\text { escolar, 3) Reflexión sobre los mecanismos de desconexión moral que usamos en } \\
\text { nuestras vidas. }\end{array}$ \\
\hline 10 & En & 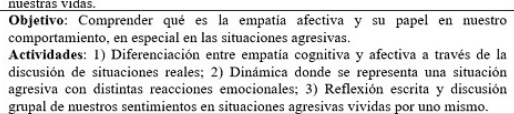 \\
\hline 11 & Revisión y cierre & $\begin{array}{l}\text { Objetivos: Valoración de las sesiones y revisión de los aprendizajes. } \\
\text { Actividades: 1) Evaluacion individual y grupal del programa; 2) Revisión de los } \\
\text { aprendizajes realizados. }\end{array}$ \\
\hline
\end{tabular}

Análisis de datos

A continuación se detallan los análisis estadísticos llevados a cabo con el programa IBM SPSS Statistics versión 23. Para comparar los resultados de las puntuaciones del grupo de intervención y del grupo control (Tablas 2 y 3 ), se usó la prueba no paramétrica de Wilcoxon. Para calcular la medida del efecto de aquellos contrastes estadísticamente significativos, se siguió a Field (2009), que propone la siguiente fórmula: $r=\mathrm{Z} / \sqrt{\mathrm{n}}$, donde $\mathrm{N}$ es el número de observaciones.

Por otro lado, para estudiar las correlaciones entre las distintas variables estudiadas, así como las correlaciones entre el cambio entre el pretest y el postest de dichas variables, se utilizó la prueba de correlaciones no paramétrica de Spearman.

\section{Resultados}

En la Tabla 2, se muestran los estadísticos descriptivos de los participantes de ambos grupos en el pretest y en el postest, así como el contraste estadístico entre ambas puntuaciones.

\section{Tabla 2}

Puntuaciones medias (y desviaciones estándar) en el pretest y postest en los grupos de intervención y control

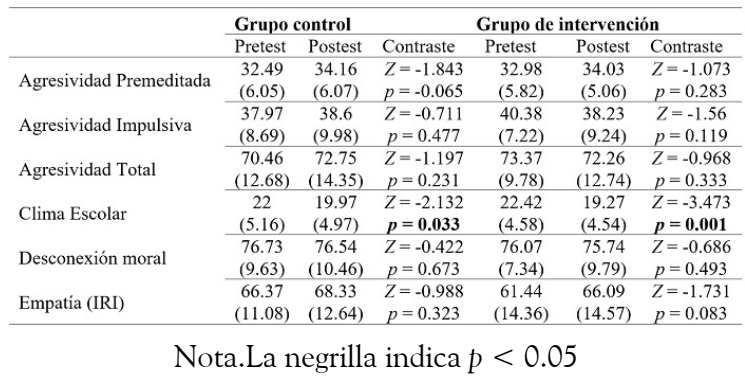

Se puede observar que la agresividad total no cambia entre el pretest y el postest en ninguno de los dos grupos de forma significativa, aunque se aprecia una disminución en el grupo de intervención y un aumento en el grupo control. En cuanto a los subcomponentes de la agresividad, la premeditada aumenta tanto en el grupo control como en el grupo de 
intervención, pero en ninguno de los dos grupos de forma significativa. Por otra parte, la agresividad impulsiva aumenta en el grupo control y disminuye en el de intervención, aunque el cambio no es significativo en ninguno de los dos casos. En relación con la empatía, esta aumenta más en el grupo de intervención que en el grupo control, siendo estos cambios no significativos en ninguno de los dos grupos. Sin embargo, las diferencias observadas en la empatía entre el pretest y el postest fueron cercanas a la significación solo en el grupo de intervención. En cuanto al clima, su puntuación disminuyó (o empeoró) de forma estadísticamente significativa tanto en el grupo de intervención como en el grupo control. Así pues, en el grupo control $r=$ $-2.132 / \sqrt{ } 64=-0.266$, mientras que en el grupo de intervención $r=-3.473 / \sqrt{ } 64=-0.434$. Por lo tanto, siguiendo a Cohen (1992), en el caso del grupo control se trataría de una medida del efecto pequeña, y en el grupo de intervención, media. En cuanto a la desconexión moral, esta se mantuvo igual después de la intervención en ambos grupos $(p>0.05)$.

Dado que se observó un cambio en el clima escolar, se investigó en qué aspectos empeoró en cada uno de los grupos (Tabla 3). En concreto, se apreció que en el grupo control solo hay una reducción significativa de la puntuación en los ítems 1.4 y 2.9. En cambio, en el grupo de intervención, hay varios ítems que se reducen de forma significativa, a saber: 1.1, 2.1, 2.2, 2.8, y 2.9. De los ítems en los que hubo cambios estadísticamente significativos, se calculó el tamaño del efecto (Grupo control: ítem 1.4, $r=0.246$; ítem 2.9, $r=0.294$, que corresponden a una medidas del efecto pequeñas; Grupo de intervención: ítem 1.1, $r=0.286$; ítem $2.1, r=0.375$; ítem 2.2, $r=0.309$; ítem 2.8, $r$ $=0.409$; ítem 2.9, $r=0.356$, que corresponden a medidas del efecto medias, excepto en el ítem 1.1 donde es pequeña.
Tabla 3

Medias (y desviaciones estándar) en los ítems del clima en el pretest y postest en función del grupo

\begin{tabular}{|c|c|c|c|c|c|c|}
\hline \multirow{2}{*}{ Ítem } & \multicolumn{3}{|c|}{ Grupo control } & \multicolumn{3}{|c|}{ Grupo de intervención } \\
\hline & Pretest & Postest & Contraste & Pretest & Postest & Contraste \\
\hline $\begin{array}{l}\text { 1.1. ¿Te encuentras bien en el } \\
\text { Instituto? }\end{array}$ & $\begin{array}{l}2.19 \\
(0.9)\end{array}$ & $\begin{array}{l}1.94 \\
(0.8)\end{array}$ & $\begin{array}{c}Z=1.79 \\
p=0.074\end{array}$ & $\begin{array}{c}2.19 \\
(0.53)\end{array}$ & $\begin{array}{c}1.87 \\
(0.82)\end{array}$ & $\begin{aligned} Z & =2.13 \\
p & =0.033\end{aligned}$ \\
\hline 1.2. ¿Las aulas son agradables? & $\begin{array}{c}1.41 \\
(0.98)\end{array}$ & $\begin{array}{c}1.37 \\
(0.61)\end{array}$ & $\begin{array}{c}Z=0.25 \\
p=0.802\end{array}$ & $\begin{array}{c}1.41 \\
(0.61)\end{array}$ & $\begin{array}{c}1.25 \\
(0.67)\end{array}$ & $\begin{array}{c}Z=1 \\
p=0.315\end{array}$ \\
\hline $\begin{array}{l}\text { 1.3. ¿Te sientes a gusto en el } \\
\text { patio? }\end{array}$ & $\begin{array}{c}2.56 \\
(0.56)\end{array}$ & $\begin{array}{c}2.5 \\
(0.67)\end{array}$ & $\begin{array}{c}Z=0.53 \\
p=0.593\end{array}$ & $\begin{array}{c}2.31 \\
(0.59)\end{array}$ & $\begin{array}{c}2.31 \\
(0.59)\end{array}$ & $\begin{array}{l}Z=0 \\
p=1\end{array}$ \\
\hline $\begin{array}{l}\text { 1.4. ¿Hay buen ambiente en el } \\
\text { centro? }\end{array}$ & $\begin{array}{c}2.2 \\
(0.061)\end{array}$ & $\begin{array}{c}1.93 \\
(0.67)\end{array}$ & $\begin{array}{c}Z=1.97 \\
p=\mathbf{0 . 0 4 9}\end{array}$ & $\begin{array}{l}2.12 \\
(0.55)\end{array}$ & $\begin{array}{c}1.97 \\
(0.54)\end{array}$ & $\begin{aligned} Z & =1.21 \\
p & =0.225\end{aligned}$ \\
\hline 2.1. En clase se dicen palabrotas & $\begin{array}{c}1.06 \\
(0.76)\end{array}$ & $\begin{array}{c}1 \\
(0.76)\end{array}$ & $\begin{array}{c}Z=0.61 \\
p=0.539\end{array}$ & $\begin{array}{c}1.06 \\
(0.72)\end{array}$ & $\begin{array}{c}0.56 \\
(0.62)\end{array}$ & $\begin{array}{c}Z=3 \\
p=0.003\end{array}$ \\
\hline 2.2. No se respetan las normas & $\begin{array}{c}1.47 \\
(0.62)\end{array}$ & $\begin{array}{c}1.25 \\
(0.67)\end{array}$ & $\begin{array}{c}Z=1.29 \\
p=0.197\end{array}$ & $\begin{array}{c}1.48 \\
(0.63)\end{array}$ & $\begin{array}{c}1.06 \\
(0.76)\end{array}$ & $\begin{array}{r}Z=2.43 \\
p=\mathbf{0 . 0 1 5}\end{array}$ \\
\hline $\begin{array}{l}\text { 2.3. Hay silencio para poder } \\
\text { trabajar }\end{array}$ & $\begin{array}{c}0.91 \\
(0.64)\end{array}$ & $\begin{array}{c}0.84 \\
(0.51)\end{array}$ & $\begin{array}{l}Z=0.58 \\
p=0.564\end{array}$ & $\begin{array}{c}1 \\
(0.62)\end{array}$ & $\begin{array}{c}0.97 \\
(0.78)\end{array}$ & $\begin{array}{l}Z=0.22 \\
p=0.83\end{array}$ \\
\hline 2.4. A menudo hay broncas & $\begin{array}{c}1.22 \\
(0.71)\end{array}$ & $\begin{array}{c}1.03 \\
(0.65)\end{array}$ & $\begin{array}{c}Z=1.33 \\
p=0.184\end{array}$ & $\begin{array}{l}1.12 \\
(0.71)\end{array}$ & $\begin{array}{c}1.19 \\
(0.78)\end{array}$ & $\begin{array}{l}Z=0.35 \\
p=0.72\end{array}$ \\
\hline $\begin{array}{l}\text { 2.5. Hay chicos poco integrados } \\
\text { que se sienten solos }\end{array}$ & $\begin{array}{c}2.13 \\
(0.72)\end{array}$ & $\begin{array}{c}2.06 \\
(0.76)\end{array}$ & $\begin{array}{c}Z=0.58 \\
p=0.564\end{array}$ & $\begin{array}{l}2.41 \\
(0.56)\end{array}$ & $\begin{array}{c}2.19 \\
(0.64)\end{array}$ & $\begin{array}{c}Z=0.71 \\
p=0.088\end{array}$ \\
\hline $\begin{array}{l}\text { 2.6. Normalmente hay más } \\
\text { orden que descontrol }\end{array}$ & $\begin{array}{c}1.06 \\
(0.91)\end{array}$ & $\begin{array}{c}0.78 \\
(0.71)\end{array}$ & $\begin{array}{l}Z=1.78 \\
p=0.074\end{array}$ & $\begin{array}{c}1.2 \\
(0.61)\end{array}$ & $\begin{array}{c}0.91 \\
(0.78)\end{array}$ & $\begin{aligned} Z & =0.74 \\
p & =0.082\end{aligned}$ \\
\hline $\begin{array}{l}\text { 2.7. Los profesores no entienden } \\
\text { a los alumnos }\end{array}$ & $\begin{array}{c}1.56 \\
(0.84)\end{array}$ & $\begin{array}{c}1.43 \\
(0.08)\end{array}$ & $\begin{array}{c}Z=0.74 \\
p=0.461\end{array}$ & $\begin{array}{c}1.59 \\
(0.91)\end{array}$ & $\begin{array}{c}1.66 \\
(0.87)\end{array}$ & $\begin{array}{l}Z=0.204 \\
p=0.838\end{array}$ \\
\hline 2.8. Los alumnos se aburren & $\begin{array}{c}0.84 \\
(0.81)\end{array}$ & $\begin{array}{l}0.78 \\
(0.75)\end{array}$ & $\begin{array}{c}Z=0.5 \\
p=0.617\end{array}$ & $\begin{array}{l}1.06 \\
(0.76)\end{array}$ & $\begin{array}{c}0.59 \\
(0.71)\end{array}$ & $\begin{aligned} Z & =3.27 \\
p & =0.001\end{aligned}$ \\
\hline $\begin{array}{l}\text { 2.9. Hay conflictos entre los } \\
\text { alumnos }\end{array}$ & $\begin{array}{c}1.94 \\
(0.44)\end{array}$ & $\begin{array}{c}1.59 \\
(0.71)\end{array}$ & $\begin{array}{c}Z=2.35 \\
p=0.019\end{array}$ & $\begin{array}{c}1.84 \\
(0.57)\end{array}$ & $\begin{array}{c}1.41 \\
(0.71)\end{array}$ & $\begin{array}{c}Z=2.85 \\
p=0.004\end{array}$ \\
\hline $\begin{array}{l}\text { 2.10. Hay conflictos entre } \\
\text { alumnos y profesores }\end{array}$ & $\begin{array}{c}1.48 \\
(0.81)\end{array}$ & $\begin{array}{c}1.43 \\
(0.84)\end{array}$ & $\begin{array}{c}Z=0.27 \\
p=0.785\end{array}$ & $\begin{array}{l}1.59 \\
(0.87)\end{array}$ & $\begin{array}{c}1.37 \\
(0.79)\end{array}$ & $\begin{aligned} Z & =1.24 \\
p & =0.021\end{aligned}$ \\
\hline
\end{tabular}

Nota.La negrilla indica $p<0.05$

$\mathrm{Al}$ estudiar las correlaciones entre la empatía, la desconexión moral y clima escolar, con la agresividad (premeditada, impulsiva y total), se encontraron las correlaciones significativas siguientes. En el pretest, la desconexión moral correlacionó negativamente con la agresividad premeditada $(r=-0.27 ; p=0.032 ; N=63)$, impulsiva $(r=-0.535 ; p<0.001 \mathrm{~N}=63)$ y total $(r=-0.526 ; p<0.001 ; N=63)$. En el postest, de nuevo, la desconexión moral correlacionó negativamente con la agresividad premeditada $(r=-0.418 ; p=0.001 ; N=60)$, impulsiva $(r=-0.444 ;<0.001 ; N=60)$ y total $(r=$ -0.463; $p<0.001 ; N=60)$. Ni la puntuación total del clima escolar ni la puntuación total de empatía correlacionaron con la agresividad, ni en el pretest ni en el postest $(p>0.05)$.

Finalmente, se estudiaron las correlaciones sobre el cambio en las puntuaciones de empatía, desconexión moral y clima escolar con el de las puntuaciones de agresividad (premeditada, impulsiva y total). Para hacerlo, se restaron las 
puntuaciones del pretest a las puntuaciones del postest, obteniendo así una puntuación de cambio para cada una de las variables mencionadas. Los resultados obtenidos muestran que solo existe una correlación (negativa) estadísticamente significativa entre el cambio en la agresividad impulsiva y el cambio en la subescala de fantasía de la empatía $(r=-0.326 ; p<0.001 ; N=61)$.

\section{Discusión}

Este estudio pretende evaluar una intervención educativa centrada en la mejora de las habilidades socioemocionales y morales del alumnado, con el fin de reducir la agresividad y mejorar la convivencia en aulas de 2. - de educación secundaria. Los resultados muestran que no hubo cambios estadísticamente significativos ni en las variables individuales estudiadas, ni tampoco en la agresividad, medidas a través de autoinformes. En cambio, se encontró una correlación entre el cambio en la agresividad impulsiva y el cambio en la subescala de fantasía de la empatía, y también correlaciones entre la desconexión moral y la agresividad, tanto en el pretest como en el postest. Además, se observó un empeoramiento del clima escolar tanto en el grupo control como en el grupo de intervención. A continuación, se comentan con detalle estos resultados.

El programa no ha cumplido su objetivo de reducir los comportamientos agresivos de los participantes. Wilson y Lipsey (2007), en una revisión de intervenciones educativas con el objetivo de prevenir la agresividad y las conductas disruptivas, encontraron que los efectos de las intervenciones eran mayores en aquellos estudiantes con alto riesgo de comportamiento agresivo. Esto podría explicar por qué no se encontraron efectos mayores en el presente estudio. Para futuras intervenciones, podría ser interesante escoger centros educativos o grupos de alumnos especialmente problemáticos, y plantear programas específicos diseñados para trabajar con los estudiantes más disruptivos. Por otra parte, aunque se evaluó la agresividad impulsiva y premeditada, no se hizo una intervención diferencial. En este sentido, las futuras intervenciones podrían centrarse en la falta de regulación de la rabia y los sesgos atribucionales hostiles en el alumnado con la agresividad impulsiva (reactiva), por un lado, y por otro, en ayudar a construir relaciones empáticas, a identificar el malestar en los demás y a balancear las metas individuales con las sociales, en el caso del alumnado con agresividad premeditada o proactiva (Bookhout et al., 2018).

En el presente estudio, no se observaron cambios significativos entre el pretest y el postest sobre la empatía. Sin embargo, el haber encontrado un aumento cercano a la significación en el grupo de intervención sugiere que un trabajo más extensivo y específico podría ser eficaz. Además, las diferencias en la empatía entre el pretest y el postest con respecto a la subescala de fantasía del IRI, están relacionadas con una disminución de la agresividad impulsiva. En este sentido, intervenir para mejorar las habilidades empáticas del alumnado de secundaria podría ser una vía para disminuir sus comportamientos agresivos. Aunque los resultados de la literatura no son del todo consistentes, la empatía afectiva parece estar relacionada con el comportamiento agresivo en la adolescencia (Lovett \& Sheffield, 2007). Las dificultades con la empatía afectiva pueden explicarse por razones como falta de respuesta al castigo o al malestar de los otros (Hughes, 2011), por dificultades en la comprensión de determinadas emociones como la rabia o la tristeza (Bookhout et al., 2018) o por dificultades de comprensión y expresión de las propias emociones (Winter, Spengler, Bermpohl, Singer, \& Kanske, 2017). Así pues, la futura investigación debería explorar con mayor profundidad el papel de la empatía afectiva en el comportamiento agresivo, así como los mecanismos a través de los cuales se puede mejorar.

La desconexión moral es un tipo de razonamiento moral que implica mecanismos (propuestos por Bandura) que permiten a las personas separarse de sus estándares morales internalizados, manteniendo un autoconcepto 
positivo y sin tener remordimientos o sentir culpabilidad (Roncero, Andreu, \& Peña, 2016). Los niños y jóvenes con una mayor desconexión moral muestran más conductas disruptivas, agresivas, de acoso y ciberacoso, mientras que la baja desconexión moral está asociada con comportamientos de defender a la víctima (Gini, Pozzoli, \& Hymel, 2014; Ortega, Sánchez, \& Menesini, 2002; Thornberg \& Jungert, 2014). Los resultados coinciden con la literatura previa, ya que se encontraron correlaciones negativas entre la desconexión moral y el comportamiento agresivo. Sin embargo, la intervención educativa no ha modificado la desconexión moral del alumnado. Es posible que sea necesario un trabajo más específico o intenso para obtener cambios. De acuerdo con el estudio de MartínezGonzález, Robles-Haydar, Amar-Amar y CrespoRomero (2016) la utilización de mecanismos de desconexión moral de los niños está relacionada con la incentivación de la violencia en las relaciones interpersonales por parte de los padres, sugiriendo la importancia de la formación de padres durante la infancia.

En cuanto al clima escolar, se constató un empeoramiento en ambos grupos, de control e intervención. La percepción del clima escolar puede haber disminuido porque, en efecto, han empeorado los elementos que lo conforman, pero los resultados también podrían indicar un toma de conciencia sobre la existencia en un mal clima en el aula. Por otra parte, en una revisión sistemática de la literatura sobre las prácticas destinadas a mejorar el clima en la enseñanza secundaria, Voight y Nation (2016) comentan que aquellas intervenciones basadas en la práctica pueden ser más adaptables a los contextos de cada profesor. En este sentido, la intervención podría haber sido implementada por los profesores, adaptando mejor las actividades a los problemas de sus aulas, y evaluando el clima a partir del consenso con el alumnado, de aquellos aspectos que se deben mejorar. En cualquier caso, hay que seguir investigando cómo mejorar el clima escolar, ya que este tiene un profundo impacto en el funcionamiento de la clase, la violencia escolar, los comportamientos problemáticos y el ajuste psicológico del alumnado (Trianes, Blanca, de la Morena, Infante, \& Raya, 2006; Wang \& Degol, 2016).

Una de las limitaciones importantes del presente estudio fue la pérdida de muestra. Es posible que aquellos participantes que se perdieron sesiones fueran los que más podrían beneficiarse de una intervención de este tipo. En segundo lugar, es posible que la intervención se hubiese beneficiado de la implicación del profesorado y de las familias. Se sabe, por ejemplo, que los programas de intervención dirigidos a mejorar las habilidades de los padres son efectivos para mejorar la capacidad de los niños de regular sus emociones y disminuir la agresividad reactiva (Bookhout et al., 2018); también, que la percepción del alumnado sobre la implicación del profesorado en las relaciones positivas de la comunidad escolar es importante para evitar la victimización den situaciones de bullying (Casas, Ortega-Ruiz, \& Del Rey, 2015). Finalmente, los contenidos implementados no incluyen la ciberagresividad. Garaigordobil y Martínez-Valderrey (2014) instauraron un programa antiacoso que incluía tanto el trabajo en situaciones de acoso presencial como en línea, y encontraron una disminución de la victimización cara a cara, así como un aumento de las conductas sociales positivas. Aunque dicho estudio no permite conocer cuál es el efecto diferencial de incluir contenidos de ciberacoso, es positivo que los adolescentes hayan podido pensar sobre cómo pueden afrontar este tipo de situaciones. Por otra parte, el programa Asegúrate, basado en la teoría del comportamiento social normativo, las habilidades de autorregulación y las creencias de los adolescentes, y diseñado para intervenir en ciberbullying, se ha mostrado efectivo no solo para reducir la prevalencia de ciberagresiones, sino también de agresiones en contextos físicos (del Rey-Alamillo, Mora-Merchán, Casas, Ortega-Ruiz, \& Elipe, 2018; del Rey, OrtegaRuiz, \& Casas, 2019). En resumen, aunque los programas de intervención en ciberbullying pueden ser herramientas efectivas para reducir la victimización, la investigación necesita aún identificar qué componentes de este tipo de 
programas son más eficaces (Gaffney, Farrington, Espelage, \& Ttofi, 2019).

\section{Conclusiones}

Varias revisiones muestran que los programas universales de aprendizaje socioemocional son eficaces para desarrollar el bienestar, el rendimiento académico y mejorar las conductas agresivas y disruptivas en la escuela, incluso seis meses después de la intervención (Domitrovich, Durlak, Staley, \& Weissberg, 2017; Durlak et al., 2011; Taylor et al., 2017). En la presente investigación, se pretendía evaluar la eficacia de un programa de tutorías que no implicara muchos recursos al centro educativo $\mathrm{ni}$ al profesorado, y que pudiera mostrar las bondades de integrar intervenciones socioemocionales y morales en sus programas docentes. A pesar de alguna tendencia positiva, los resultados no han satisfecho los objetivos propuestos, pero sí que aportan algunas reflexiones. En primer lugar, sobre la importancia de la frecuencia y la intensidad de las sesiones; es decir, la dosis necesaria para que los programas de intervención sean eficaces y eficientes. Los programas incluidos en las revisiones consultadas no realizan ningún estudio detallado sobre esta variable. Se entiende que las sesiones necesarias pueden diferir según la población y el contexto; por ejemplo, Durlak et al. (2011) afirman que los estudiantes más jóvenes pueden necesitar más sesiones que los mayores, pero no consta que las revisiones muestren qué horquilla de sesiones pueden mejorar significativamente las intervenciones. Por último, es evidente que muchos otros factores pueden haber influido en la falta de eficacia de la intervención de este estudio (la integración en el proyecto educativo de la escuela, la aplicación a contextos no académicos, el uso de las TIC para implicar al alumnado en el proyecto, etc.). En este sentido, se requiere conocer cuáles son los elementos centrales de los programas de intervención socioemocionales y morales y cómo se deben trabajar, para poder obtener mejoras significativas que hagan rentable su aplicación en el difícil contexto educativo de la educación secundaria.

\section{Agradecimientos}

Queremos agradecer al Institut-escola Francesc Cambó i Batlle de Verges y al Institut Caldes de Malavella su colaboración en el presente trabajo. Damos las gracias también a Naila Freixes y Gerard Rigau por su inestimable colaboración en la realización del proyecto. Este trabajo de investigación no ha recibido ningún tipo de apoyo financiero específico de instituciones públicas, privadas o sin ánimo de lucro.

\section{Referencias}

Andreu, J. M. (2010). Cuestionario de Agresividad Premeditada e Impulsiva en Adolescentes. Madrid: Tea Ediciones.

Arribas, D., \& Corral, S. (2011). BAS2, Escalas de aptitudes intelectuales. Madrid: TEA Ediciones.

Bandura, A., Barbaranelli, C., Caprara, G. V., \& Pastorelli, C. (1996). Mechanisms of moral disengagement in the exercise of moral agency. Journal of Personality and Social Psychology, 71(2), 364-374. https://doi.org/ 10.1037/0022-3514.71.2.364

Blair, R. J. R. (2003). Did Cain fail to represent the thoughts of Abel before he killed him? The relationship between theory of mind and aggression. En B. Repacholi \& V. Slaughter (Eds.), Individual differences in theory of mind. Implications for typical and atypical development (pp. 144-171). Nueva York: Psychology Press.

Bookhout, M. K., Hubbard, J. A., \& Moore, C. C. (2018). Emotion regulation. En J. E. Lochman \& W. Matthys (Eds.), The Wiley handbook of disruptive and impulsecontrol disorders (pp. 221-236). Hoboken, NJ: Wiley.

Card, N., Stucky, B., Sawalani, G., \& Little, T. (2008). Direct and indirect aggresion during childhood and adolescence: A meta-analytic review of gender 
differences, intercorrelations, and relations to maladjustment. Child Development, 79(5), 1185-1229. https://doi.org/10.1111/j $.1467-8624.2008 .01184 . x$

Carrasco, M. Á., Delgado, B., Barbero, M. I., Holgado, F. P., \& del Barrio, M. V. (2011). Propiedades psicométricas del Interpersonal Reactivity Index (IRI) en población infantil y adolescente española. Psicothema, 23(4), 824-831. Recuperado de http://www.psicothema.com/PDF/3962.pdf

Carroll, A., McCarthy, M., Houghton, S., Sanders O'Connor, E., \& Zadow, C. (2018). Reactive and proactive aggression as meaningful distinctions at the variable and person level in primary schoolaged children. Aggressive Behavior, 44(5), 431-441. https://doi.org/10.1002/ab.21763

Casas, J. A., Ortega-Ruiz, R., \& del Rey, R. (2015). Bullying: The impact of teacher management and trait emotional intelligence. British Journal of Educational Psychology, 85(3), 407-423. https://doi.org/ 10.1111/bjep. 12082

Cohen, J. (1992). A power primer. Psychological Bulletin, 112, 155-159. https://doi.org/10.10 37/0033-2909.112.1.155

Collell, J., \& Escudé, C. (2004). La convivència en els centres: elements per a la intervenció preventiva de la violència entre iguals a l'escola. Recuperado de http://www.xtec.cat/sgfp/li cencies/200203/resums/jcollell.html

Conley, C. S. (2015). SEL in higher education. En J. A. Durlak, C. E. Domitrovich, R. P. Weissberg \& T. P. Gullotta (Eds.), Handbook of social and emotional learning: research and practice (pp. 197-212). Nueva York: Guilford.

da Silva, J. L., de Oliveira, W. A., de Mello, F. C. M., de Andrade, L. S., Bazon, M. R., \& Silva, M. A. I. (2017). Anti-bullying interventions in schools: A systematic literature review. Ciencia $\mathbb{E}$ Saude Coletiva, 22(7), 2329-2340. https://doi.org/10.1590/ 1413-81232017227.16242015

Davis, M. (1980). A multidimensional approach to individual differences in empathy. JSAS Catalog of Selected Documents in Psychology,
10, 85. Recuperado de https://www.uv.es/ friasnav/Davis_1980.pdf

del Rey-Alamillo, R., Mora-Merchán, J. A., Casas, J. A., Ortega-Ruiz, R., \& Elipe, P. (2018). Programa «Asegúrate»: efectos en ciberagresión y sus factores de riesgo. Comunicar, 26(56), 39-48. https://doi.org/1 0.3916/C56-2018-04

del Rey, R., Ortega-Ruiz, R., \& Casas, J. A. (2019). Asegúrate: An intervention program against cyberbullying based on teachers' commitment and on design of its instructional materials. International Journal of Environmental Research and Public Health, 16(3), 434. https://doi.org/10.3390/ijerph1 6030434

DePaoli, J. L., Atwell, M., \& Bridgeland, J. (2017). Ready to lead: A national principal survey on how social and emotional learning can prepare children and transform schools. Washington, DC: Civic Enterprises and Hart Research Associates for CASEL.

Domitrovich, C. E., Durlak, J. A., Staley, K. C., \& Weissberg, R. P. (2017). Socialemotional competence: An essential factor for promoting positive adjustment and reducing risk in school children. Child Development, 88(2), 408-416. https://doi.or g/10.1111/cdev.12739

Durlak, J. A., Weissberg, R. P., Dymnicki, A. B., Taylor, R. D., \& Schellinger, K. B. (2011). The impact of enhancing students' social and emotional learning: A meta-analysis of school-based universal interventions. Child Development, 82(1), 405-432. https://doi.or $\mathrm{g} / 10.1111 / \mathrm{j} .1467-8624.2010 .01564 . \mathrm{x}$

Elias, M. J. (2009). Social-emotional and character development and academics as a dual focus of educational policy. Educational Policy, 23(6), 831-846. https://doi.org/10.11 77/0895904808330167

Elias, M. J., Parker, S. J., Kash, V. M., Weissberg, R. P., \& O'Brien, M. U. (2008). Social and emotional learning, moral education, and character education: A comparative analysis and a view toward convergence. En L. P. Nucci \& D. Narvaez (Eds.), 
Handbook of moral and character education (pp. 248-266). Nueva York: Routledge.

Field, A. P. (2009) Discovering statistics using SPSS: and sex and drugs and rock ' $n$ ' roll (3rd ed.). London, UK: Sage publications.

Gaffney, H., Farrington, D. P., Espelage, D. L., \& Ttofi, M. M. (2019). Are cyberbullying intervention and prevention programs effective? A systematic and meta-analytical review. Aggression and Violent Behavior, 45, 134-153. https://doi.org/10.1016/j.avb.201 8.07 .002

Garaigordobil, M., \& Martínez-Valderrey, V. (2014). Efecto del Cyberprogram 2.0 sobre la reducción de la victimización y la mejora de la competencia social en la adolescencia. Revista de Psicodidáctica, 19(2), 289-305. Recuperado de http://www.redalyc.org/arti culo.oa?id=17531400003

Gini, G., Pozzoli, T., \& Hymel, S. (2014). Moral disengagement among children and youth: A meta-analytic review of links to aggressive behavior. Aggressive Behavior, 40 (1), 56-68. https://doi.org/10.1002/ab.21 502

Greenberg, M. T., Weissberg, R. P., O’Brian, M. U., Zins, J. E., Fredericks, L., Resnik, H., \& Elias, M. J. (2003). Enhancing schoolbased prevention and youth development through coordinate social, emotional and academic learning. American Psychologist, 58(6-7), 466-474. https://doi.org/10.1037/0 003-066x.58.6-7.466

Hawker, D. S., \& Boulton, M. J. (2000). Twenty years' research on peer victimization and psychosocial maladjustment: A metaanalytic review of cross-sectional studies. The Journal of Child Psychology and Psychiatry and Allied Disciplines, 41(4), 441-455. https://doi.org/10.1111/1469-761 0.00629

Herrenkohl, T., Kosterman, R., Mason, W., \& Hawkins, J. (2007). Youth violence trajectories and proximal characteristics of intimate partner violence. Violence and Victims, 22, 259-74. https://doi.org/10.1891 1088667007780842793
Hughes, C. (2011). Social understanding and social lives: From toddlerhood through to the transition to school. Londres: Psychology Press.

Lovett, B. J., \& Sheffield, R. A. (2007). Affective empathy deficits in aggressive children and adolescents: A critical review. Clinical Psychology Review, 27(1), 1-13. https://doi.o $\mathrm{rg} / 10.1016 /$ j.cpr.2006.03.003

Mahoney, J. L., Durlak, J. A., \& Weissberg, R. P. (2018). An update on social and emotional learning outcome research. Phi Delta Kappan, 100(4), 18-23. https://doi.or $\mathrm{g} / 10.1177 / 0031721718815668$

Martínez-González, M. B., Robles-Haydar, C. A., Amar-Amar, J. J., \& Crespo-Romero, F. A. (2016). Crianza y desconexión moral en infantes: su relación en una comunidad vulnerable de Barranquilla. Revista Latinoamericana de Ciencias Sociales, Niñez y Juventud, 14(1), 315-330. https://d oi.org/10.11600/1692715x.14121011214

Matalí, J. (Coord.). (2016). Adolescentes con trastornos de comportamiento. ¿Cómo podemos detectarlos? ¿Qué se debe hacer? Barcelona: Hospital Sant Joan de Déu.

Olweus, D. (1991). Bully/victim problems among schoolchildren: Basic facts and effects of a school based intervention program. En D. J. Pepler \& K. H. Rubin (Eds.), The development and treatment of childhood aggression(pp. 411-448). Hillsdale, NJ: Erlbaum.

Olweus, D., Limber, S. P., \& Breivik, K. (2019). Addressing specific forms of bullying: A large-scale evaluation of the Olweus bullying prevention program. International Journal of Bullying Prevention, 1(1), 70-84. h ttps://doi.org/10.1007/s42380-019-00009-7

Ortega, R., Del Rey, R., \& Gómez, P. (2002). Estrategias educativas para la prevención de la violencia: mediación y diálogo. Madrid: Cruz Roja Juventud.

Ortega, R., Sánchez, V., \& Menesini, E. (2002). Violencia entre iguales y desconexión moral: un análisis transcultural. Psicothema, 14(Supl. 1), 
37-49. Recuperado de http://www.psicothe ma.com/psicothema.asp? $\mathrm{id}=3473$

O'Toole, S. E., Monks, C. P., \& Tsermentseli, S. (2017). Executive function and theory of mind as predictors of aggressive and prosocial behavior and peer acceptance in early childhood. Social Development, 26(4), 907-920. https://doi.org/10.1111/sode.1223 1

Salmivalli, C., Kärnä, A., \& Poskiparta, E. (2011). Counteracting bullying in Finland: The KiVa program and its effects on different forms of being bullied. International Journal of Behavioral Development, 35(5), 405-411. https://doi.or g/10.1177/0165025411407457

Rieffe, C., Broekhof, E., Kouwenberg, M., Faber, J., Tsutsui, M. M., \& Güroğlu, B. (2016). Disentangling proactive and reactive aggression in children using selfreport. European Journal of Developmental Psychology, 13(4), 439-451. https://doi.org/ 10.1080/17405629.2015.1109506

Roncero, D., Andreu, J. M., \& Peña, M. E. (2016). Procesos cognitivos distorsionados en la conducta agresiva y antisocial en adolescentes. Anuario de Psicología Jurídica, 26(1), 88-101. https://doi.org/10.1016/j.apj .2016 .04 .002

Taylor, R. D., Oberle, E., Durlak, J. A., \& Weissberg, R. P. (2017). Promoting positive youth development through school-based social and emotional learning interventions: A meta-analysis of followup effects. Child Development, 88(4), 1156-1171. https://doi.org/10.1111/cdev.1 2864

Trianes, M. V., Blanca, M. J., De la Morena, L., Infante, L., \& Raya, S. (2006). Un cuestionario para evaluar el clima social del centro escolar. Psicothema, 18(2), 272-277. Recuperado de http://www.psicothema.co $\mathrm{m} /$ psicothema.asp? $\mathrm{id}=3209$

Thornberg, R., \& Jungert, T. (2014). School bullying and the mechanisms of moral disengagement. Aggressive Behavior, 40(2), 99-108. https://doi.org/10.1002/ab.21509
Voight, A., \& Nation, M. (2016). Practices for improving secondary school climate: A systematic review of the research literature. American Journal of Community Psychology, 58(1-2), 174-191. https://doi.org/10.1002/a jcp. 12074

Wang, M. T., \& Degol, J. L. (2016). School climate: A review of the construct, measurement, and impact on student outcomes. Educational Psychology Review, 28(2), 315-352. https://doi.org/10.1007/s10 648-015-9319-1

Weir, H., \& Kaukinen, C. (2015). Delinquent effects of childhood exposure to violent victimization: A latent longitudinal class analysis. Violence and Crime in the Family: Patterns, Causes, and Consequences, 255-284. https://doi.org/10.1108/s1530-35 3520150000009012

Weissberg, R. P., Durlak, J. A., Domitrovich, C. E., \& Gullotta, T. P. (2015). Social and emotional learning: Past, present, and future. En J. A. Durlak, C. E. Domitrovich, R. P. Weissberg \& T. P. Gullotta (Eds.), Handbook of social and emotional learning: Research and practice (pp. 3-19). Nueva York: Guilford Press.

Wiglesworth, M., Lendrum, A., Oldfield, J., Scott, A., ten Bokkel, I., Tate, K., \& Emery, C. (2016). The impact of trial stage, developer involvement and international transferability on universal social and emotional learning programme outcomes: A meta-analysis. Cambridge Journal of Education, 46(3), 347-376. https://doi.org/1 0.1080/0305764X.2016.1195791

Wilson, S. J., \& Lipsey, M. W. (2007). Schoolbased interventions for aggressive and disruptive behavior: Update of a metaanalysis. American Journal of Preventive Medicine, 33(2), S130-S143. https://doi.org /10.1016/j.amepre.2007.04.011

Winter, K., Spengler, S., Bermpohl, F., Singer, T., \& Kanske, P. (2017). Social cognition in aggressive offenders: Impaired empathy, but intact theory of mind. Scientific Reports, 
Francesc Sidera, Carles Rostan, Jordi Collell, et al.

7(1), 670. https://doi.org/10.1038/s41598-0

17-00745-0

\section{Notas}

* Artículo de investigación. 